\title{
Establishment of Methylation-Specific PCR for the Mouse p53 Gene
}

\author{
Ryuji Okazaki, ${ }^{1}$ Akira Ootsuyama, ${ }^{1}$ Yasuhiro Yoshida, ${ }^{2}$ and Toshiyuki Norimura ${ }^{1}$ \\ ${ }^{1}$ Department of Radiation Biology and Health, School of Medicine, University of Occupational and Environmental Health, \\ 1-1 Iseigaoka Yahatanishi-ku, Kitakyushu 807-8555, Japan \\ ${ }^{2}$ Department of Immunology and Parasitology, School of Medicine, University of Occupational and Environmental Health, \\ 1-1 Iseigaoka Yahatanishi-ku, Kitakyushu 807-8555, Japan \\ Correspondence should be addressed to Ryuji Okazaki, ryuji-o@med.uoeh-u.ac.jp
}

Received 30 March 2011; Revised 8 September 2011; Accepted 19 September 2011

Academic Editor: George A. Calin

Copyright ( $) 2011$ Ryuji Okazaki et al. This is an open access article distributed under the Creative Commons Attribution License, which permits unrestricted use, distribution, and reproduction in any medium, provided the original work is properly cited.

Methylation-specific PCR (MSP) of the mouse p53 gene has not yet been reported. We searched the CpG islands, sequenced the bisulfited DNA, and designed PCR primers for methylation and unmethylation sites. DNA from a young mouse produced a strong PCR product with the unmethylated primer and a weaker band with the methylated primer. DNA from an old mouse produced bands of similar intensities with both primers. In radiation-induced tumors, DNA from an old mouse yielded similar bands with both types of primers. We suggest that MSP is a valuable technique for the epigenetic study of the mouse p53 gene.

\section{Introduction}

A number of specific loci have been described as becoming hypermethylated during aging or carcinogenesis [1]. Most remaining CpG sites are normally methylated in adult cells. Transcriptional inactivation by cytokine methylation at promoter CpG islands of tumor suppressor genes is thought to be an important mechanism in human carcinogenesis [2]. In half of all primary human cancer cells, p53 is the most commonly mutated tumor suppressor gene [3]. Moreover, p73, a p53 homologue, has been shown to be hypermethylated in leukemia [4]. Using human cancer cells, Herman and coworkers [5] first described methylation-specific PCR (MSP), which can rapidly assess the methylation status of virtually any group of $\mathrm{CpG}$ sites. The MSP primer sequences for the human p53 gene have been identified in medulloblastomas [6], human gliomas [7], and hepatocellular carcinoma [8]. While there are many mouse experiments, the MSP primer sequences for the mouse p53 gene have yet to be reported. It is thought that it helps a future study very much to establish MSP technique. In this study, to establish MSP for this gene, we analyzed a CpG site in the mouse p53 gene and designed the corresponding primer sequences.

\section{Materials and Methods}

2.1. Experimental Animals. Mice carrying a disrupted, nonfunctional p53 gene $\left(p 53^{-/-}\right)$were derived from homologous recombination in an embryonic stem cell line from 129/SvJ mice as described previously $[9,10]$. Wild type mice of the parental inbred strain were used as controls for the $p 53^{+/+}$ mice. $p 53^{+/-}$mice were obtained by crossing male $p 53^{-/-}$ mice with female $p 53^{+/+}$mice. The experimental protocols were approved by the Ethics Review Committee for Animal Experimentation of the University of Occupational and Environmental Health, Japan (Kitakyushu, Japan).

2.2. Irradiation. $\mathrm{A}^{90} \mathrm{Sr}^{90} \mathrm{Y}$ disk delivered a beta ray source of $1.85 \mathrm{GBq}$ at $15 \mathrm{~Gy} / \mathrm{min}$. The backs of seven-week-old mice $\left(p 53^{+/+}\right.$and $\left.p 53^{+/-}\right)$were irradiated with beta rays three times a week until the appearance of a tumor. These mice received 5 Gy/day.

2.3. Isolation of Genomic DNA. Genomic DNA from the spleen, liver, kidney, and tumor was isolated using a QuickGene DNA (Fujifilm Holdings Corporation, Tokyo, Japan) whole blood kit according to the manufacturer's instructions. 


\begin{abstract}
Exon4 TCATCACCTCACTGCATGGACGATCTGTTGCTGCCCCAGGATGTTGAGGAGTTTTTTGAAGGCC CAAGTGAAGCCCTCCGAGTGTCAGGAGCTCCTGCAGCACAGGACCCTGTCACCGAGACCCCTG GGCCAGTGGCCCCTGCCCCAGCCACTCCATGGCCCCTGTCATCTTTTGTCCCTTCTCAAAAAAC TTACCAGGGCAACTATGGCTTCCACCTGGGCTTCCTGCAGTCTGGGACAGCCAAGTCTGTTATG TGCACGGTGAGTGGGCCCCGGGGAGTTGTCTTTCGTGTGACCTTTCAATGCCTCTTTTTCTCTGTCTA GATTTGGGGGTTCCTCTTCAGCCTGTAGACTGTGCCTCAGAGTTTAGTTTTGCCCTGAACCTTTTTAG ССТCTTCTCCTTTCTCTCATATTCTCTGCATCTCTCCAGGGGACGTGGAACTCTCTTCCCTCACATTCC TTTCTTGGCTTTTGAAAATAATCTTCTGAAGCCAAGCACAGAGGCGTGTGCTGTAGGCCCAGCTGCT AGAGACAGTTGAGGCAGGATTGCTTAAGGCCAGCCTGAGCATGGAAGTAAGACCCCTTCTCACCAA AAACAAAAACAGCCCAGTGGAGTGACACACACCTGTAGCTCCAGCACTGGGGAGGCCAAAGTGGG AGGGACAAAAGTTCGAGGCCATCTCTGACTACACAGAGAGGTGCCCTCTCAAAAAAAGAGAAGTC AGGTGTGTTGGCCATCTCTGTGAGTTCGAGGCCAGCCTGGTCTACAAAGTGAGTTCCAGGACAGCC AGGGCTACATAGAGAAACCCTGTCTCGAAAAACACAGAAAGGGAGGAAGAAGGAAAGGTCCCAGT CCTCTCTTTGCTGGCTGCCCTCTGCCACTGCGAGGGCGTCCAATGGTGCTTGGACAATGTGTTTCATT AGTTCCCCACCTTGACACCTGATCGTTACTCGGCTTGTCCCCGACCTCCGTTCTCTCTCCTCTCTTCC

Exon5 AGTACTCTCCTCCCCTCAATAAGCTATTCTGCCAGCTGGCGAAGACGTGCCCTGTGCAGTTGTG GGTCAGCGCCACACCTCCAGCTGGGAGCCGTGTCCGCGCCATGGCCATCTACAAGAAGTCACA GCACATGACGGAGGTCGTGAGACGCTGCCCCCACCATGAGCGCTGCTCCGATGGTGATGGTAA GCCCTCAACACCGCCTGTGGGGTTAGGACTGGCAGCCTCCCATCTCCCGGCTTCTGACTTATTCTTG

Exon6 CTCTTAGGCCTGGCTCCTCCCCAGCATCTTATCCGGGTGGAAGGAAATTTGTATCCCGAGTATCT GGAAGACAGGCAGACTTTTCGCCACAGCGTGGTGGTACCTTATGAGCCACCCGAGGTCTGTAAT TTTGTTTTGGTTTGTGCGTCTTAGAGACAGTTGACTCCAGCCTAGACTGATGTTGACTTTCTAGCAAC CCGTTTGCCTCACCCTCCTGAGTGCTAGCTAGGCTTAGAGGTGCAAGCTGCCGTGCCCAGCCCAGGG TCTACTTTAACAGCAGTCTCTGGGAGAAGGGGCTTCCCCATCCAGGCGGGAAATAGAGACGCTGAG TCCGGTTCCCTCCCATGCTAAGCAAGTGTTGGGCCCCACAGCTCCAGCAGGTGTGCCGAACAGGTG GAATATCCCTACTCTACAACTAAAACTGAAACTTATTAGAGGCTATAGCCAGCCATTCCCGGCTGCT GCAGGTCACCTGTAGTGAGGTAGGGAGCGACTTCACCTGGATCCTGTGTCTTCCCCCAGGCCGGCT

Exon7 CTGAGTATACCACCATCCACTACAAGTACATGTGTAATAGCTCCTGCATGGGGGGCATGAACCG CCGACCTATCCTTACCATCATCACACTGGAAGACTCCAG
\end{abstract}

(a)

\begin{abstract}
TTTGTTTCGAAAAATATAGAAAGGGAGGAAGAAGGAAAGG TTTTAGTTTTTTTTTTGTTGGTTGTTTTTTGTTATTGCGAGGG CGTTTAATGGTGTTTGGATAATGTGTTTTATTAGTTTTTTATTT TGATATTTGATCGTTATTCGGTTTGTTTTCGATTTTCGTTTTTT TTTTTTTTTTTTAGTATTTTTTTTTTTTTAATAAGTTATTTTGTT AGTTGGCGAAGACGTGTTTTGTGTAGTTGTGGGTTAGCGTTA TATTTTTAGTTGGGAGTCGTGTTCGCGTTATGGTTATTTATAA GAAGTTATAGTATATGACGGAGGTCGTGAGACGTTGTTTTTA TTATGAGCGTTGTTTCGATGGTGATGGTAAGTTTTTAATATCG TTTGTGGGGTTAGGATTGGTAGTTTTTTATTTTTCGGTTTTTG ATTTATTTTTGTTTTTAGGTTTGGTTTTTTTTTAGTATTTTATTC GGGTGGAAGGAAATTTGTATTTCGAGTATTTGGAAGATAGGT AGATTTTTCGTTATAGCGTGGTGGTATTTTATGAGTTATTCGA GGTTTGTAATTTTGTTTTGGTTTGTGCGTTTTAGAGATAGTTG ATTTTAGTTTAGATTGA
\end{abstract}

(b)

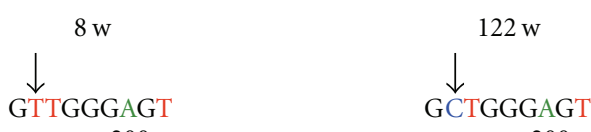

200
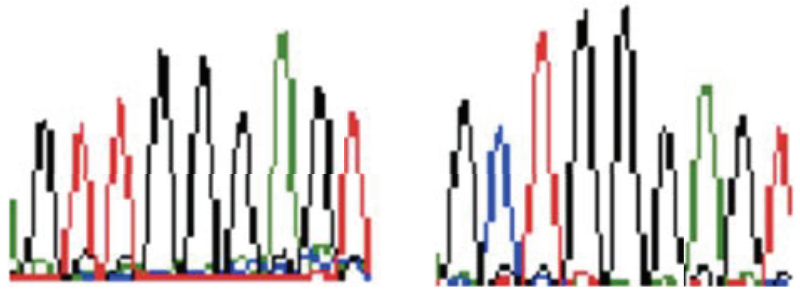

(c)

Figure 1: (a) CpG islands of the mouse p53 gene from exon 4 to exon 9. Underlined sequences indicate CpG islands that were searched using Methyl Primer Express Software v1.0. The cytosine (C) surrounded by a rectangle is the CpG site. (b) The sequence of the CpG islands after bisulfite conversion. The boxed area indicates the forward and reverse primer sets. Underline sequences refer to exons 5 and 6 . (c) Electropherograms of PCR products after subcloned plasmid DNA in 8-week-old and 122-week-old p53/+ mice. In 8-week-old mice, a "C" was converted to a " $T$ ". In 122-week-old mice, a "C" was retained as a " $\mathrm{C}$ ".

2.4. Bisulfite Conversion. Approximately, $1 \mu \mathrm{g}$ of DNA was treated with sodium bisulfite using an EpiTect Bisulfite kit (Qiagen, Hilden, Germany) according to the manufacturer's instructions. Briefly, the DNA, bisulfite mix, and DNA Protect buffer were mixed together. The bisulfite conversion thermal cycling conditions were as follows: $99^{\circ} \mathrm{C}$ for $5 \mathrm{~min}$, $60^{\circ} \mathrm{C}$ for $25 \mathrm{~min}, 99^{\circ} \mathrm{C}$ for $5 \mathrm{~min}, 60^{\circ} \mathrm{C}$ for $85 \mathrm{~min}, 99^{\circ} \mathrm{C}$ for $5 \mathrm{~min}$, and $60^{\circ} \mathrm{C}$ for $175 \mathrm{~min}$. Finally, the bisulfite-converted DNA was purified on a spin column and eluted with $20 \mu \mathrm{L}$ of EB buffer.

2.5. Amplification of PCR Product. PCR primers were designed using Methyl Primer Express Software v1.0 (Applied
Biosystems Inc. Foster City, CA, USA). PCR primer sets included sense (5'-ATC GTT ATT CGG TTT GTT TTC-3') and antisense ( $5^{\prime}$-CAC GAC CTC CGT CAT ATA CT-3') primers. Thermal cycling conditions were as follows: 1 cycle at $95^{\circ} \mathrm{C}$ for $10 \mathrm{~min} ; 30$ cycles at $94^{\circ} \mathrm{C}$ for $15 \mathrm{~s}, 55^{\circ} \mathrm{C}$ for $30 \mathrm{~s}$, and $72^{\circ} \mathrm{C}$ for $30 \mathrm{~s} ; 1 \mathrm{cycle}$ at $72^{\circ} \mathrm{C}$ for $10 \mathrm{~min}$.

2.6. Ligation, Transformation, and Sequence. PCR products were inserted into a pcDNA 3.1/V5-His-TOPO vector by TOPO cloning technology. The reaction was mixed gently and incubated for 5 minutes at room temperature. Two microliters of this reaction mixture were then added to a tube of TOP10 competent E. coli and mixed gently. After incubating 
TABle 1: Primer sequences for p53 MSP analysis.

\begin{tabular}{ccc}
\hline & & Primer sequence $\left(5^{\prime}-3^{\prime}\right)$ \\
\hline $\mathrm{U}^{1}$ & $\mathrm{~F}^{3}$ & ATC GTT ATT CGG TTT GTT TTC \\
& $\mathrm{R}^{4}$ & CGA ACA CGA CTC CCA ACT AA \\
$\mathrm{M}^{2}$ & $\mathrm{~F}$ & ATC GTT ATT CGG TTT GTT TTC \\
& $\mathrm{R}$ & CGA ACA CGA CTC CCA GCT AA \\
\hline
\end{tabular}

${ }^{1} \mathrm{U}$ : un-methylated sequence; ${ }^{2} \mathrm{M}$ : methylated sequence; ${ }^{3} \mathrm{~F}$ : forward sequence; ${ }^{4} \mathrm{R}$ : reverse sequence.

on ice for 30 minutes, the cells were heat shocked for $30 \mathrm{sec}$ onds at $42^{\circ} \mathrm{C}$ without shaking. Tubes were immediately transferred back to the ice, and $250 \mu \mathrm{L}$ of room temperature medium was added. After incubation at $37^{\circ} \mathrm{C}$ for 30 minutes, reaction mixtures were spread onto a prewarmed ampicillin plate and incubated overnight at $37^{\circ} \mathrm{C}$. Colonies were picked and cultured overnight in LB medium containing $50 \mu \mathrm{g} / \mathrm{mL}$ ampicillin. We isolated the plasmid DNA using a miniprep kit (Qiagen), and its sequence was analyzed using both the T7 primer and reverse sequencing primers.

2.7. Sequence Analysis. DNA sequencing was performed with a BigDye terminator v3.1 cycle sequencing kit (Applied Biosystems Inc.) according to the manufacturer's instructions using the T7 sequencing primer (Invitrogen, Carlsbad, CA, USA) and the sense primer $5^{\prime}$-TAA TAC GAC TCA CTA TAG GG-3'. Thermal cycling conditions included 1 cycle at $96^{\circ} \mathrm{C}$ for $1 \mathrm{~min}$ and 25 cycles at $96^{\circ} \mathrm{C}$ for $10 \mathrm{~s}, 50^{\circ} \mathrm{C}$ for $5 \mathrm{~s}$, and $60^{\circ} \mathrm{C}$ for $4 \mathrm{~min}$. The DNA sequencing reactions were purified using the BigDye XTerminator purification kit (Applied Biosystems Inc.). The DNA sequencing reactions in 96 well plates were run on a 3130/3130xl genetic analyzer (Applied Biosystems Inc.), and resulting sequences were analyzed with sequencing analysis software (Applied Biosystems Inc.).

2.8. Methylation Specific PCR (MSP). We searched for CpG islands in the $\mathrm{p} 53$ gene and designed PCR primer sets using Methyl Primer Express Software v1.0 (Applied Biosystems Inc.). PCR amplification was performed using an EpiTect MSP kit (Qiagen). Thermal cycling conditions were as follows: 1 cycle at $95^{\circ} \mathrm{C}$ for $10 \mathrm{~min} ; 35$ cycles at $94^{\circ} \mathrm{C}$ for $15 \mathrm{~s}$, $50^{\circ} \mathrm{C}$ for $1 \mathrm{~min}$ and $72^{\circ} \mathrm{C}$ for $30 \mathrm{~s} ; 1$ cycle at $72^{\circ} \mathrm{C}$ for $10 \mathrm{~min}$.

\section{Results and Discussion}

3.1. Mapping of DNA Methylation Patterns in a Cp G Islands of the Mouse p53 Gene and the Sequence of Bisulfited DNA. We searched the mouse p53 gene from exon 4 to 9 for a CpG islands using Methyl Primer Express Software v1.0 (Figure 1(a); Applied Biosystems Inc.) and found one located from intron 5 to intron 7, spanning 619 base pairs. The cytosine (C) surrounded with a rectangle is the specific CpG site (Figure 1(a)). This is the first description of a CpG island for the p53 gene in normal mouse tissues. Using the same software, the $\mathrm{CpG}$ island was searched with simulated bisulfiteconverted DNA sequences. The resulting sequences were the same as the sequences that were analyzed by the genetic analyzer in this study.

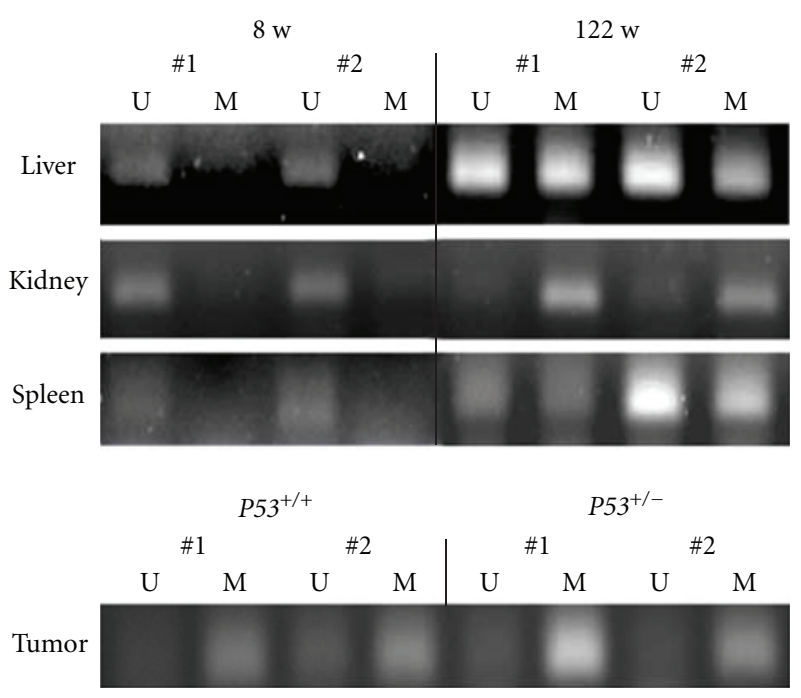

FIGURE 2: MSP analysis. Primer sets used for amplification are designated as unmethylated (U) and methylated (M). Liver, kidney, and spleen samples were from two 8-week-old mice and two 122week-old $p 53^{+/+}$mice. Tumors were induced by a ${ }^{90} \mathrm{Sr}-{ }^{90} \mathrm{Y}$ beta ray in $p 53^{+/+}$and $p 53^{+/-}$mice.

Several candidates for PCR primer sets were given by the software, and the suitable set, determined after many rounds of PCR, included a methylated base. The best sets for p53unmethylation and p53-methylation are surrounded by a rectangle in Figure 1(b) and given in Table 1. Cytosine (C) of GCT (Alanine) in exon 5 was converted to a thymidine (T) after bisulfite treatment in young mice, which did not occur in old mice after the same treatment (Figures 1(b), 1(c)).

3.2. MSP Analysis. In all organs (i.e., liver, kidney, and spleen) of 8-week-old mice, we obtained a strong PCR product using the unmethylated primer and weaker band with the methylated primer. Alternatively, in liver and spleen from 122-week-old mice, the DNA was amplified to similar PCR products with both the unmethylated and methylated primers (Figure 2).

In radiation-induced tumors from $p 53^{+/+}$mice and $p 53^{+/-}$mice, amplified DNA yielded similar bands with both the methylated and unmethylated primers (Figure 2) except in one tumor from a $p 53^{+/-}$mouse, which showed a strong band with the former and a weaker band with the latter.

We have previously shown data for MSP in the $\mathrm{p} 53$ gene [11] where p53 methylation increased with age in the spleen after irradiation at a young age. After DNA damage is induced by intra- and/or extracellular processes, DNA methylation is known to increase with increasing age [12]. In this study, we have shown how p53 methylation in the spleen and liver relates to age and that p53 methylation was found in all radiation-induced tumors in mice. Finally, it is interesting to note that methylation increases in tumor suppressor genes in human tumors as well [13], including the p53 gene [6-8].

In this study, we established MSP for the mouse p53 gene, and this is the first description. Unfortunately, we were only able to find one base (C) that was methylated, observing 
bands of similar intensity using both unmethylated and methylated primers. Therefore, there is still room for improvement in this MSP method. However, we could show MSP technique for mouse p53. The technique is so simple and easy. It is thought that the width of the methylation study will progress in future by MSP technique for the mouse p53 gene having been established.

\section{Conflict of Interests}

The authors declare no conflict of interests.

\section{Acknowledgments}

The authors thank Mr. Toshiki Iwamoto and Miss Emiko Nakama, who are medical students in UOEH, for technical assistance. This work was supported in part by UOEH Grant for Advanced Research (H18-3), UOEH Research Grant for Promotion of Occupational Health from the University of Occupational and Environmental Health, Japan (no. 909), and a Grants-in-Aid for Scientific Research (C) from the Japan Society for the Promotion of Science (no. 19510061).

\section{References}

[1] S. F. Gilbert, "Ageing and cancer as diseases of epigenesis," Journal of Biosciences, vol. 34, no. 4, pp. 601-604, 2009.

[2] J. P. Issa, "CpG island methylator phenotype in cancer," Nature Reviews Cancer, vol. 4, no. 12, pp. 988-993, 2004.

[3] P. Hainaut and M. Hollstein, "p53 and human cancer: the first ten thousand mutations," Advances in Cancer Research, vol. 77, pp. 81-137, 2000.

[4] P. G. Corn, S. J. Kuerbitz, M. M. van Noesel et al., "Transcriptional silencing of the p73 gene in acute lymphoblastic leukemia and Burkitt's lymphoma is associated with $5^{\prime} \mathrm{CpG}$ island methylation," Cancer Research, vol. 59, no. 14, pp. 3352$3356,1999$.

[5] J. G. Herman, J. R. Graff, S. Myohanen, B. D. Nelkin, and S. B. Baylin, "Methylation-specific PCR: a novel PCR assay for methylation status of CpG islands," Proceedings of the National Academy of Sciences of the United States of America, vol. 93, no. 18, pp. 9821-9826, 1996.

[6] M. C. Fruhwald, M. S. O’Dorisio, Z. Dai et al., "Aberrant promoter methylation of previously unidentified target genes is a common abnormality in medulloblastomas-implications for tumor biology and potential clinical utility," Oncogene, vol. 20, no. 36, pp. 5033-5042, 2001.

[7] V. J. Amatya, U. Naumann, M. Weller, and H. Ohgaki, “TP53 promoter methylation in human gliomas," Acta Neuropathologica, vol. 110, no. 2, pp. 178-184, 2005.

[8] C. Zhang, X. Guo, G. Jiang et al., "CpG island methylator phenotype association with upregulated telomerase activity in hepatocellular carcinoma," International Journal of Cancer, vol. 123, no. 5, pp. 998-1004, 2008.

[9] Y. Gondo, K. Nakamura, K. Nakao et al., "Gene replacement of the 553 gene with the lacZ gene in mouse embryonic stem cells and mice by using two steps of homologous recombination," Biochemical and Biophysical Research Communications, vol. 202, no. 2, pp. 830-837, 1994.
[10] T. Norimura, S. Nomoto, M. Katsuki, Y. Gondo, and S. Kondo, "p53-dependent apoptosis suppresses radiation-induced teratogenesis," Nature Medicine, vol. 2, no. 5, pp. 577-580, 1996.

[11] R. Okazaki, A. Ootsuyama, H. Kakihara et al., "Dynamics of delayed p53 mutations in mice given whole-body irradiation at 8 weeks," International Journal of Radiation Oncology Biology Physics, vol. 79, no. 1, pp. 247-254, 2011.

[12] D. M. Shin, M. Kucia, and M. Z. Ratajczak, "Nuclear and chromatin reorganization during cell senescence and aging-a mini-review," Gerontology, vol. 57, pp. 76-84, 2010.

[13] M. Esteller, "Relevance of DNA methylation in the management of cancer," Lancet Oncology, vol. 4, no. 6, pp. 351-358, 2003. 

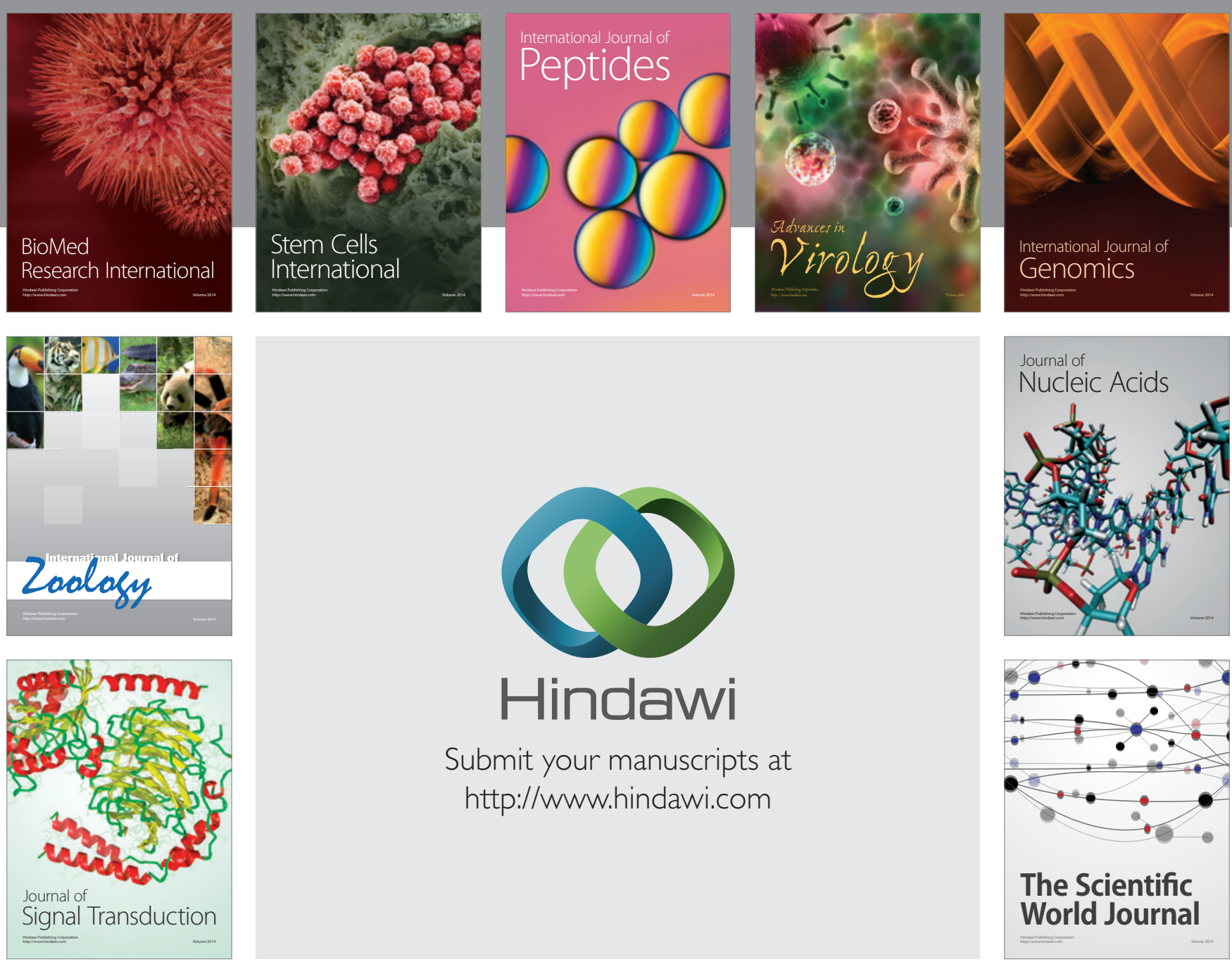

Submit your manuscripts at

http://www.hindawi.com
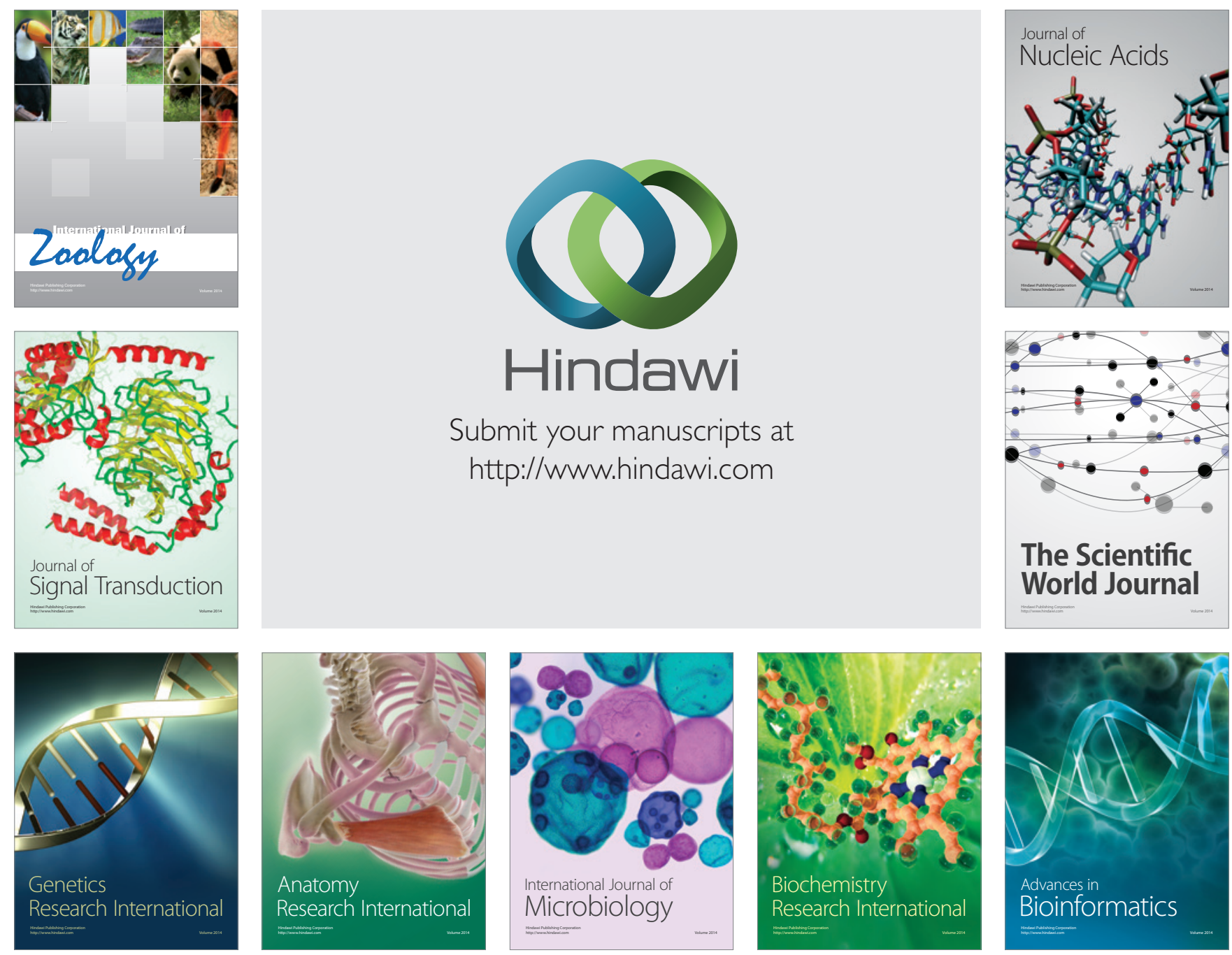

The Scientific World Journal
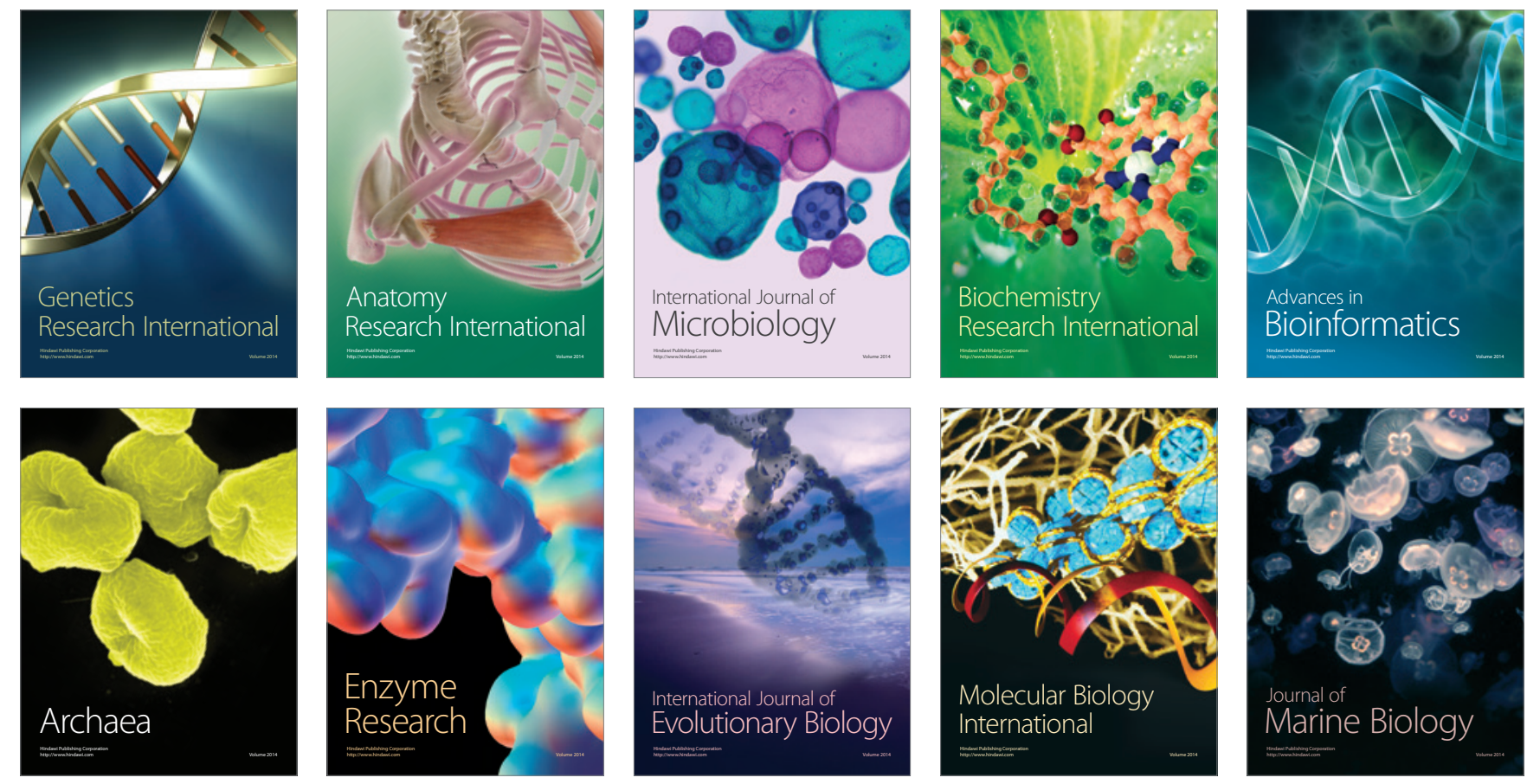